\title{
Receptividad al aprendizaje de grupos nominales en inglés según el nivel MCERL
}

\author{
Ana Luz Rubio Moreda \\ Universidad Politécnica de Madrid, España \\ Artículo recibido 27 de octubre de 2014, aceptado 30 de marzo de 2015, versión final recibida 6 de junio de \\ 2015 \\ DOI: http://dx.doi.org/10.5565/rev/jtl3.603
}

\begin{abstract}
Resumen
El objetivo de este estudio es comprobar cómo la enseñanza explícita de los grupos nominales (GN) en inglés científico-técnico (ICT), facilita su adquisición en alumnos de ingeniería españoles de acuerdo con su nivel de competencia del Marco Común Europeo de Referencia de las Lenguas (MCERL).Se aplicó una metodología basada en 'noticing' con alumnos de diferentes niveles MCERL. Los resultados se analizaron estadísticamente concluyendo que para asimilar dicho rasgo lingüístico es preciso superar el umbral de usuario independiente B1, con un resultado en progresión ascendente al pasar a usuario competente $\mathrm{C} 1 \mathrm{y}$ descendente al aumentar la complejidad del GN.
\end{abstract}

Palabras clave: grupos nominales, noticing, competencia gramatical, ICT, MCERL

\begin{abstract}
The aim of this study is to find out whether the explicit teaching of nominal groups (NG) in English for Science and Technology (EST), helps its acquisition by Spanish engineering students with different English level according to the Common European Framework of Reference for Languages (CEFRL). For this purpose, a 'noticing' methodological approach was designed and tested with a sample of different CERFL level students. Results were statistically analysed to check on its efficiency, reaching the conclusion that in order to acquire such linguistic feature a threshold level of independent user B1 is required, with evidence of a progressive improvement as users reach $\mathrm{C} 1$ competent level, but decreasing as the NG becomes more complex.
\end{abstract}

Keywords: nominal groups, noticing, grammar competence, EST, CEFRL levels

\section{Résumé}

L'objectif de cette étude est de vérifier comment l'enseignement spécifique des groupes nominaux (GN) en Anglais technico-scientifique facilite leur acquisition avec des étudiants en ingénierie espagnols à partir d'un certain niveau de compétence du Européen Commun de Référence pour les Langue, (CECRL). On a utilisé une application méthodologique expérimentale basée sur un «noticing» avec des étudiants de différents niveaux du CECRL. Les résultats ont été analysés statistiquement afin de vérifier leur efficacité, arrivant à la conclusion que pour assimiler ce trait linguistique il faut dépasser le seuil d'utilisateur indépendant B1, avec un résultat en progression ascendante en passant à utilisateur compétent $\mathrm{C} 1$ et descendante en augmentant la complexité du GN.

Mots clés: groupes nominaux, enseignement intensif, Anglais technico-scientifique, CECRL 


\section{Introducción}

El objetivo de este trabajo es comprobar si los alumnos españoles de ingeniería con diferente niveles del Marco Común Europeo de Referencia de las Lenguas (MCER) (Consejo de Europa, 2001) asimilan una estructura sintáctica inexistente en su L1, los grupos nominales (GNs) simples y complejos. Para ello se ha hallado el nivel de inglés del Marco y se ha comprobado el conocimiento y uso de los GNs con un pre-test, tanto en traducción directa como en inversa. A través de un post-test y mediando un proceso intensivo de enseñanzaaprendizaje se han comparado los resultados para comprobar si existe un nivel de competencia lingüística requerida para adquirir el uso de los GNs.

La presencia recurrente de GNs en el inglés científico técnico (ICT) se destaca como rasgo lingüístico distintivo por varios autores: Swales (1985), Trimble (1985), Huckin y Olsen (1991) Halliday y Martin (1993), Alcaraz (2000), Dudley-Evans (2001) y Cademártori, Parodi y Venegas (2006), entre otros. Y si bien diversos estudios se dedican a la enseñanza de la nominalización en inglés a alumnos de L1 española en ICT, ninguno analiza la relación existente entre el aprendizaje de esta estructura y el nivel de inglés del MCERL de los usuarios, objetivo de este trabajo.

Partiendo de la base de que el GN es la estructura lingüística que presenta mayor dificultad para los alumnos de ingeniería españoles en relación con el dominio de otros rasgos lingüísticos propios del ICT (Rubio, 2015) y su formación en español es diferente a la construcción sintáctica en inglés (Bocanegra Valle, 2007; Durán Escribano \& Rubio, 2015), su enseñanza constituye un reto para docentes y lingüistas.

Los GNs, entendidos como grupos de palabras colocados en "caravana", pueden producir "monstruos incomprensibles" (Norman, 1999, p. 57) que nos alertan de la necesidad de equipar al estudiante con herramientas que ayuden a comprenderlos. Martínez y Hurtado (2003, p. 15) afirman que se necesita dotar al alumno de ciertas "subcompetencias lingüísticas para poder procesar determinados aspectos del ICT siguiendo las instrucciones y realizando las actividades prescritas por los profesores". Ellis (1984) sostiene que el profesor debe intentar que los alumnos sean conscientes de las características específicas de la L2 practicando aquellos elementos gramaticales diferenciadores en relación con su L1 para llegar a un aprendizaje eficaz. Son estas premisas las que han inspirado el planteamiento didáctico contenido en este estudio ya que cada vez que un alumno se encuentra con un GN debe realizar ciertos pasos cognitivos con una serie consideraciones semánticas, sintácticas y estilísticas que debe adquirir para llegar a alcanzar la competencia lingüística requerida en el 
contexto académico en que utiliza el idioma. Esto le permitirá aplicar las reglas léxicogramaticales necesarias para poder entenderlo, sabiendo que el nombre premodificado en inglés es un conjunto que puede constar de tantas notas conceptuales como número de premodificadores contenga.

En el desarrollo de la docencia enfocada al aprendizaje de esta estructura sintáctica, el punto de partida es el reconocimiento consciente (noticing) de la existencia del rasgo objeto de estudio, tal y como lo concibe Schmidt "detection with awareness within selective attention” (2001, p. 3). Según este autor, la atención focalizada constituye un paso previo para la asimilación de un rasgo lingüístico distinto en L2: “noticing is the necessary and sufficient condition for converting input into intake" (Schmidt, 2001, p. 129). Esta fase se considera de vital importancia con alumnos de ingeniería para despertar en ellos la conciencia lingüística de que esta estructura es recurrente en ICT y así facilitar su comprensión y uso posterior (Durán Escribano, 2004, p. 186).

Giovannini, Martín Peris, Rodríguez y Simón (1996) apoyan la fase de identificación de los GNs basada en patrones gramaticales, al proponer una tipología de actividades para la enseñanza de L2 que comienza con los ejercicios de sensibilización gramatical. Sólo así se podrán explicar las reglas necesarias para su comprensión y ejercitación incluyendo los distintos tipos de GNs y relacionándolos con patrones gramaticales en la L1. Además, si "la práctica de las habilidades lingüísticas constituye el principal medio para alcanzar la competencia comunicativa" (Pastor Cesteros, 2004, p. 189), hemos de orientar la didáctica hacia aquellas destrezas que favorezcan el entendimiento de las relaciones entre los diferentes elementos del GN; es decir, se debe facilitar el aprendizaje mediante una combinación de adquisición consciente y una práctica de tareas, como señala Bocanegra Valle (2007, p. 77) cuando afirma que "nominal compounding demands awareness and conscious reflection. Instruction and dedicated learning may contribute to these”.

En el contexto del ICT, Huckin y Olsen (1991) y Trimble (1985) afirman que el significado de las relaciones entre las diferentes unidades de un GN suele ser de finalidad, composición, principio y modo de operación, forma, tamaño, localización, nombre del creador o referencia. Según los autores mencionados, sólo si se conocen estas relaciones, será posible comprender el significado completo del sintagma nominal.

En la misma línea se manifiestan Brown, Malmkjaer y Williams (1996), Downing (1997) y Girju, Moldovan, Tatu, y Antohe (2005) quien además añade que el contexto es necesario para la correcta decodificación de los GNs "nominal compounds (NCs) have implicit semantic relations... and the interpretation of NCs can be highly context-dependent", Bellaterra Journal of Teaching \& Learning Language \& Literature. 8.3 (Aug-Sept 2015) ISSN 2013-6196 
(pp. 479-480). Breeze (2012) reconoce que el contacto directo de los alumnos con textos auténticos de su especialidad es un incentivo básico en la didáctica de la escritura académica. Por esto, en la propuesta metodológica empleada, se han utilizado textos de la especialidad de los alumnos, donde los GNs aparecen en un contexto de ingeniería eléctrica.

\section{Metodología y Etapas del proceso de enseñanza-aprendizaje}

Los participantes en el estudio son alumnos de la Universidad Politécnica de Madrid (UPM) de la especialidad de Ingeniería Eléctrica, matriculados en la asignatura obligatoria de Inglés para la comunicación, correspondiente a la titulación de Ingeniero Industrial. La muestra la constituyen los 50 alumnos que asistieron a clase y completaron todas las tareas y pruebas requeridas. El nivel MCER de los participantes se encontraba entre los niveles A2 (7), B1 (21), B2 (14) y C1 (8), baremado mediante el Oxford Placement Test que se aplicó al comienzo de la docencia general de la asignatura.

Para evaluar el grado de conocimiento de los GNs, se confeccionó un test de traducción directa e inversa con frases extraídas de las revistas de especialidad en las que había presencia de GNs de entre 2 y 4+ elementos. El test contaba con 27 GNs, tanto en traducción directa como inversa (Tabla 1). Se ha considerado GN el formado por un núcleo (nombre) principal y todos los premodificadores (no adjetivos), que aparecen como $\mathrm{X}$ en la tabla.

Tabla 1. GNs utilizados en el test inicial y final en traducción directa e inversa

\begin{tabular}{lccc}
\hline & & T.Directa & T.Inversa \\
\hline GN de 2 elementos: & X+NOMBRE & 4 & 5 \\
\hline GN de 3: & X+X+NOMBRE & 10 & 15 \\
\hline GN de 4+: & $\mathrm{X}+\mathrm{X}+\mathrm{X}+$ NOMBRE & 13 & 7 \\
\hline TOTAL GN: & & 27 & 27 \\
\hline
\end{tabular}

Se trabajaron diez artículos (de entre 7,000 y 10,000 palabras) procedentes de publicaciones recientes del IEEE (Institute of Electrical and Electronics Engineers), seleccionados con el asesoramiento del Departamento de Ingeniería Eléctrica de la Escuela Técnica Superior de Ingenieros Industriales (ETSII) de la UPM.

Previa a la aplicación didáctica propiamente dicha, se aplicó a los alumnos el test inicial de traducción directa e inversa de frases con presencia de GNs de diferente grado de complejidad. En ningún caso se mencionó la necesidad de prestar atención a la estructura objeto de análisis.

El proceso de enseñanza-aprendizaje se llevó a cabo durante cuatro semanas (tres horas por semana) y comenzó trabajando en tres etapas sobre los artículos de especialidad seleccionados: 
1. Lectura intensiva (input) de un solo artículo por alumno para asimilar su contenido.

2. Lectura detallada del artículo en la que cada alumno subrayó los GNs, para fijar su atención en la presencia de los GNs, (noticing).

3. Análisis de los GNs según dos criterios :

a) número de premodificadores no adjetivos $(2,3,4+)$ y

b) categoría gramatical de los premodificadores (nombre, verbo, preposición, adverbio).

En este punto se explicaron las reglas gramaticales que regulan la formación de los GNs orientando a los alumnos hacia lo siguiente:

a) Límites del GN, identificando primero el núcleo y después los premodificadores.

b) Recuento y análisis de la categoría gramatical de los premodificadores.

c) Comprensión del orden lógico semántico de los premodificadores.

d) Interpretación de los GNs y posibles traducciones al español.

Una vez completadas las fases de enseñanza-aprendizaje y para comparar los resultados contrastándolos con los obtenidos inicialmente, se volvió a pasar a los alumnos el mismo test inicial tanto en traducción directa como inversa y se anotaron los resultados.

La tabla 2 resume las tareas que se realizaron, el tipo de sesión en la que se desarrollaron (presencial o virtual, utilizando la plataforma Aula Web) y la duración de las mismas.

Tabla 2. Organización de las tareas de enseñanza-aprendizaje

\begin{tabular}{|c|c|c|c|}
\hline ESTRATEGIA & TAREA & TIPO DE SESIÓN & DURACIÓN \\
\hline Búsqueda & $\begin{array}{l}\text { Identificar GN en el } \\
\text { texto dado }\end{array}$ & $\begin{array}{l}\text { Presencial } \\
\text { Aula Web }\end{array}$ & Hora y media \\
\hline $\begin{array}{l}\text { Focalización de la } \\
\text { atención }\end{array}$ & $\begin{array}{l}\text { Técnicas de } \\
\text { identificación de GN }\end{array}$ & Presencial & Hora y media \\
\hline Clasificación & $\begin{array}{l}\text { Ordenar GN por } \\
\text { categorías de } \\
\text { premodificadores }\end{array}$ & No presencial & Dos horas \\
\hline Guía del proceso & $\begin{array}{l}\text { Comprobar las } \\
\text { selecciones y su } \\
\text { clasificación }\end{array}$ & $\begin{array}{l}\text { No presencial } \\
\text { Aula Web }\end{array}$ & Una hora \\
\hline Análisis del trabajo & $\begin{array}{l}\text { Definir las } \\
\text { clasificaciones y dar } \\
\text { explicaciones } \\
\text { gramaticales }\end{array}$ & Presencial & Hora y media \\
\hline $\begin{array}{l}\text { Practica de ejercicios } \\
\text { de aplicación }\end{array}$ & $\begin{array}{l}\text { Ejercicios } \\
\text { (español/inglés) }\end{array}$ & $\begin{array}{l}\text { No presencial } \\
\text { Aula Web }\end{array}$ & Dos horas \\
\hline Clarificación de dudas & $\begin{array}{l}\text { Exposición y resolución } \\
\text { de problemas planteados } \\
\text { durante la ejecución del } \\
\text { ejercicio }\end{array}$ & Presencial & Una hora \\
\hline Corrección & Corrección & $\begin{array}{c}\text { No Presencial } \\
\text { Aula Web }\end{array}$ & Una hora \\
\hline Evaluación & Aplicación de los tests & Presencial & Dos horas \\
\hline
\end{tabular}




\section{finales}

El desarrollo de las fases segunda y tercera del proceso de enseñanza-aprendizaje se centra en el análisis de los GNs. Para que el modelo fuera el mismo y se facilitara la clasificación de los GNs, se entregó una plantilla a cada alumno que debía completar con los datos de su artículo distinguiendo y clasificando los GNs según el número de elementos que lo formaban.

El ejercicio se comenzó en clase, explicando que sólo se buscaban GNs premodificados por otros nombres, verbos (participios de pasado y presente), y frases preposicionales. Se ofrecieron algunos ejemplos de GNs en cada artículo analizado para que los alumnos procedieran a la ejecución del ejercicio.

Mi labor como profesora consistió en revisarlo y enviar individualmente por la plataforma web de la asignatura una primera corrección. Se les dio un día para que volcaran los resultados definitivos utilizando la plantilla y se colgó una versión revisada de cada artículo para que los alumnos comprobaran su trabajo y anotaran cualquier duda. Todos los alumnos tuvieron acceso a la selección de GNs de todos los artículos.

La siguiente sesión en el aula consistió en el análisis de los GNs teniendo en cuenta las diferentes categorías gramaticales que se encontraron en la traducción directa, señaladas en el apartado anterior. La presencia de los diferentes elementos que forman el GN fue el punto de partida para explicar las relaciones lingüístico-semánticas que se establecen entre los diferentes premodificadores y facilitar así a los alumnos la comprensión de los GNs en sus artículos.

A continuación se comentaron las clasificaciones de los GNs en cada artículo y se corrigieron los errores centrando la atención en los ejemplos concretos y continuando con la realización de ejercicios propuestos por el profesor en la plataforma virtual.

Al término de esta fase se pidió a los alumnos que proporcionaran textos de su propia especialidad con ejemplos de GNs, clasificándolos según el número de premodificadores que los formaban.

La aplicación didáctica terminó cuando los alumnos realizaron el test final

\section{Análisis estadístico de los resultados y discusión}

Los resultados de los dos tests (inicial y final) en sus dos versiones (traducción directa e inversa) fueron transferidos a una matriz de recolección de datos y tratados estadísticamente mediante el análisis de la varianza factorial (ANOVA). Las respuestas fueron dicotómicas, es decir, bien traducido (B) o mal traducido (M). La tabla 3 contiene una muestra con los 
resultados de tres alumnos por cada nivel del MCER, en la que los números 2, 3 y 4+ hacen referencia al número de elementos de cada GN y la fila siguiente, al total de aciertos posibles. La primera columna corresponde a la traducción directa y la segunda (negrita) a la inversa. Las dos últimas columnas contienen los totales de aciertos de cada alumno en el conjunto de los GNs.

Tabla 3. Muestra de los resultados de los tests inicial y final de GNs TEST INICIAL

TEST FINAL

\begin{tabular}{|c|c|c|c|c|c|c|c|c|c|}
\hline \multirow{2}{*}{\multicolumn{2}{|c|}{\begin{tabular}{|l|}
$\mathrm{N}^{\circ}$ elementos en $\mathrm{GN}$ \\
$\mathrm{NN}^{\circ}$ posibles aciertos \\
\end{tabular}}} & \multicolumn{2}{|l|}{2} & \multicolumn{2}{|l|}{3} & \multicolumn{2}{|l|}{$4+$} & \multicolumn{2}{|l|}{ Total } \\
\hline & & 4 & 5 & 10 & 15 & 13 & 7 & 27 & 27 \\
\hline & A2 & 2 & 1 & 3 & 5 & 1 & 0 & 6 & 6 \\
\hline & A2 & 3 & 2 & 2 & 4 & 2 & 0 & 7 & 6 \\
\hline & A2 & 2 & 2 & 2 & 5 & 1 & 0 & 5 & 7 \\
\hline & B1 & 3 & 3 & 7 & 7 & 5 & 1 & 15 & 11 \\
\hline & B1 & 3 & 3 & 6 & 6 & 4 & 0 & 13 & 9 \\
\hline & B1 & 3 & 4 & 6 & 7 & 6 & 0 & 15 & 11 \\
\hline & B2 & 3 & 4 & 7 & 7 & 6 & 2 & 16 & 13 \\
\hline & B2 & 3 & 4 & 7 & 6 & 6 & 2 & 16 & 12 \\
\hline & B2 & 3 & 4 & 8 & 7 & 7 & 3 & 18 & 14 \\
\hline & $\mathrm{C} 1$ & 4 & 4 & 8 & 9 & 9 & 3 & 20 & 17 \\
\hline & $\mathrm{C} 1$ & 4 & 4 & 9 & 10 & 8 & 4 & 20 & 19 \\
\hline & $\mathrm{C} 1$ & 4 & 4 & 9 & 9 & 8 & 4 & 20 & 18 \\
\hline
\end{tabular}

\begin{tabular}{|l|l|l|l|l|l|l|l|l|l|}
\hline & 2 & & 3 & & $4+$ & & Total & \\
\hline & 4 & $\mathbf{5}$ & 10 & $\mathbf{1 5}$ & 13 & $\mathbf{7}$ & 27 & $\mathbf{2 7}$ \\
\hline A2+ & 3 & $\mathbf{2}$ & 6 & $\mathbf{7}$ & 4 & $\mathbf{2}$ & 13 & $\mathbf{1 1}$ \\
\hline A2+ & 3 & $\mathbf{3}$ & 6 & $\mathbf{6}$ & 5 & $\mathbf{3}$ & 14 & $\mathbf{1 2}$ \\
\hline A2+ & 4 & $\mathbf{3}$ & 6 & $\mathbf{7}$ & 4 & $\mathbf{4}$ & 16 & $\mathbf{1 4}$ \\
\hline B1 & 4 & $\mathbf{4}$ & 8 & $\mathbf{9}$ & 7 & $\mathbf{5}$ & 19 & $\mathbf{1 8}$ \\
\hline B1+ & 4 & $\mathbf{5}$ & 8 & $\mathbf{9}$ & 8 & $\mathbf{5}$ & 20 & $\mathbf{1 9}$ \\
\hline B1+ & 4 & $\mathbf{4}$ & 8 & $\mathbf{9}$ & 6 & $\mathbf{4}$ & 18 & $\mathbf{1 7}$ \\
\hline B2- & 4 & $\mathbf{4}$ & 9 & $\mathbf{1 2}$ & 11 & $\mathbf{5}$ & 24 & $\mathbf{2 1}$ \\
\hline B2 & 4 & $\mathbf{5}$ & 9 & $\mathbf{1 2}$ & 11 & $\mathbf{5}$ & 24 & $\mathbf{2 2}$ \\
\hline B2 & 4 & $\mathbf{5}$ & 9 & $\mathbf{1 2}$ & 11 & $\mathbf{5}$ & 24 & $\mathbf{2 2}$ \\
\hline C1 & 4 & $\mathbf{5}$ & 9 & $\mathbf{1 3}$ & 12 & $\mathbf{5}$ & 25 & $\mathbf{2 3}$ \\
\hline C1 & 4 & $\mathbf{5}$ & 10 & $\mathbf{1 5}$ & 13 & $\mathbf{6}$ & 27 & $\mathbf{2 6}$ \\
\hline C1+ & 4 & $\mathbf{5}$ & 10 & $\mathbf{1 4}$ & 12 & $\mathbf{6}$ & 26 & $\mathbf{2 5}$ \\
\hline
\end{tabular}

En el análisis estadístico, se tomaron como variables los cuatro niveles MERC (A2, B1, B2 y C1) correspondientes a los alumnos participantes y la complejidad de los diferentes GNs (2, 3 y 4+ elementos). He de mencionar que la complejidad se refiere exclusivamente al número de elementos premodificadores del GN y no a la categoría gramatical de los mismos. Para hallar la diferencia de las respuestas de los alumnos de esos cuatro niveles respecto a cada GN y poder aceptar que los valores obtenidos en el estudio fueran significativos y estadísticamente fiables, se fijó el valor probabilístico $\mathrm{P} \leq 0,05$ (Tablas 4 y 5).

Tabla 4. Análisis de la varianza en el nivel de mejora en traducción directa

\begin{tabular}{|c|c|c|c|c|c|}
\hline Variables & um of Squares & Df & Mean Square & F-Ratio & P-Value \\
\hline A:DIFICULTAD & 30,9364 & 2 & 15,4682 & 14,76 & 0,0000 \\
\hline B:NIVEL MCER & 24,122 & 3 & 8,04066 & 7,67 & 0,0001 \\
\hline INTERACCIÓN A y B & 50,1512 & 6 & 8,35853 & 7,98 & 0,0000 \\
\hline \multicolumn{6}{|c|}{ Tabla 5. Análisis de la varianza en el nivel de mejora en traducción inversa } \\
\hline${ }_{\text {P-Value }}^{\text {Variables }}$ & \multicolumn{2}{|c|}{ Sum of Squares } & \multicolumn{2}{|c|}{ Df Mean Square } & F-Ratio \\
\hline A:DIFICULTAD & 155,712 & 2 & 77,8562 & 75,65 & 0,0000 \\
\hline B:NIVEL MCER & 6,2725 & 3 & 2,09085 & 2,03 & 0,1123 \\
\hline INTERACCIÓN A y B & 18,3867 & 6 & 3,06445 & 2,98 & 0,0091 \\
\hline
\end{tabular}


Las tablas muestran que el valor $\mathrm{P} \leq 0,05$ establecido para aceptar los resultados con un índice de fiabilidad del 95\%, se cumple en todos los casos. Observamos una excepción en la variable "nivel MCER" con un valor $\mathrm{P}=0,11$ debido a la inesperado mejora de resultados del nivel A2 en la traducción inversa de GNs de 4+ elementos. En las conclusiones se ofrecen posibles razones que pueden justificar este dato.

En la presentación de los resultados se han diferenciado dos bloques: traducción directa y traducción inversa, analizándolos por grado de dificultad (número de elementos que forman el GN) y por nivel MCER (A2, B1, B2 y C1). También se han comparado los resultados obtenidos en los tests inicial (I) y final (F), obteniendo lo que denominamos nivel de mejora.

\section{Traducción Directa. Análisis por grado de dificultad}

La tabla 6 muestra la puntuación media sobre 10 en el test inicial (I), por grado de dificultad de los GNs, así como la mejora obtenida, diferencia con el test final (F).

Tabla 6. Medias por dificultad en el test I de traducción directa y de mejoras en test $\mathrm{F}$

\begin{tabular}{ccrc} 
DIFICULTAD & NoALUMNOS & $\begin{array}{c}\text { PUNTUACIÓN } \\
\text { MEDIA TEST I }\end{array}$ & $\begin{array}{c}\text { MEJORA MEDIA } \\
\text { EN TEST F }\end{array}$ \\
\hline 2 & 50 & 7,7381 & 1,59226 \\
\hline 3 & 50 & 5,76042 & 2,32292 \\
\hline $4+$ & 50 & 3,89766 & 2,80714 \\
\hline
\end{tabular}

Se observa cómo la puntuación media de los resultados del total del grupo en traducción directa de los GNs de 4+ elementos es la más baja $(3,89)$, no llegando a la media de 5 que sí se alcanza en los GNs de 3 elementos y se supera en los GNs de 2 con 7,73 puntos.

La mejora media del grupo, basada en las diferencias de los resultados entre el test I y el test $\mathrm{F}$ de cada alumno, indica que el conjunto de los 50 alumnos mejoran en traducción directa de los GNs. Teniendo en cuenta su grado de dificultad, vemos que en los GNs de 2 elementos la mejora es de 1,59. Esta va aumentando progresivamente en los GNs de mayor dificultad, ya que el conocimiento inicial de los GNs complejos era menor. Así, el incremento en la puntuación en los GNs de 3 elementos es de 2,32, y en los de 4+ elementos de 2,80 puntos. La figura 1 muestra esta información gráficamente. 


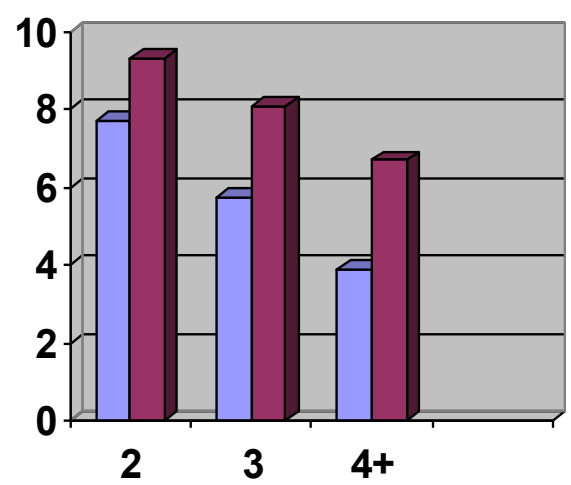

\section{$\square$ Inicial \\ Final}

Figura 1. Comparación entre Test I y test F por grado de dificultad en traducción directa

\section{Traducción Directa. Análisis por nivel MCER}

La tabla 7 muestra las puntuaciones globales medias en el test inicial por nivel MCER de los alumnos, sin distinción del grado de dificultad.

Tabla 7. Puntuación media por nivel MCER en test I en traducción directa

\begin{tabular}{ccc} 
NIVEL & NoALUMNOS & PUNTUACIÓN GLOBAL MEDIA \\
\hline A1 & 7 & 3,163 \\
\hline B1 & 21 & 5,21001 \\
\hline B2 & 14 & 6,61355 \\
\hline C1 & 8 & 8,20833
\end{tabular}

Para valorar las diferencias entre los distintos niveles en el test I de traducción directa y su comportamiento respecto al grado de dificultad, es necesario conocer las medias de los participantes de cada nivel MCER, así como el incremento de mejora (Tabla 8).

Tabla 8. Medias por dificultad y por nivel en test I de traducción directa y de mejora en test $\mathrm{F}$

\begin{tabular}{llrcc} 
DIFICULTAD & $\begin{array}{l}\text { NIVEL } \\
\text { MCER }\end{array}$ & N $^{\text {A ALUMNOS }}$ & $\begin{array}{c}\text { PUNTUACIÓN } \\
\text { MEDIA TEST I }\end{array}$ & $\begin{array}{c}\text { MEJORA MEDIA } \\
\text { EN TEST F }\end{array}$ \\
\hline 2 & A2 & 7 & 6,07143 & 2,14286 \\
\hline 2 & B1 & 21 & 7,38095 & 1,90476 \\
\hline 2 & B2 & 14 & 7,50 & 2,32143 \\
\hline 2 & C1 & 8 & 10,00 & 0,0 \\
\hline 3 & & & 2,42857 & 3,71429 \\
\hline 3 & A2 & 7 & 4,95238 & 2,66667 \\
\hline 3 & B1 & 21 & 7,28571 & 1,78571 \\
\hline 3 & B2 & 14 & 8,375 & 1,1250 \\
\hline $4+$ & C1 & 8 & & \\
\hline $4+$ & & & 0,98901 & 2,74286 \\
\hline $4+$ & A2 & 7 & 3,2967 & 2,11429 \\
\hline $4+$ & B1 & 21 & 5,05495 & 3,12143 \\
\hline
\end{tabular}

La última columna de la tabla 8 indica que el mayor índice de mejora se encuentra en los alumnos de nivel A2 con los GNs 3 elementos (3,71 puntos), seguidos por el grupo C1 
con GNs de 4+ elementos (3,25 puntos) y del nivel B2 con GNs 4+ elementos (3,12 puntos). En el resto, el incremento en la puntuación se sitúa alrededor de 2 puntos, con la notable excepción de los alumnos con nivel C1 en GNs 2 elementos, que no mejoran porque partían de 10 puntos, y en GNs 3 elementos, cuya mejora es de 1,12 puntos ya que partían de una puntuación de 8,37.

Como resumen del análisis por nivel MCER, se presentan dos diagramas de barras en los que observamos los resultados de los tests de traducción directa, por grado de dificultad de los GNs y por niveles MCER, en el test inicial (Figura 2) y en el test final (Figura 3).

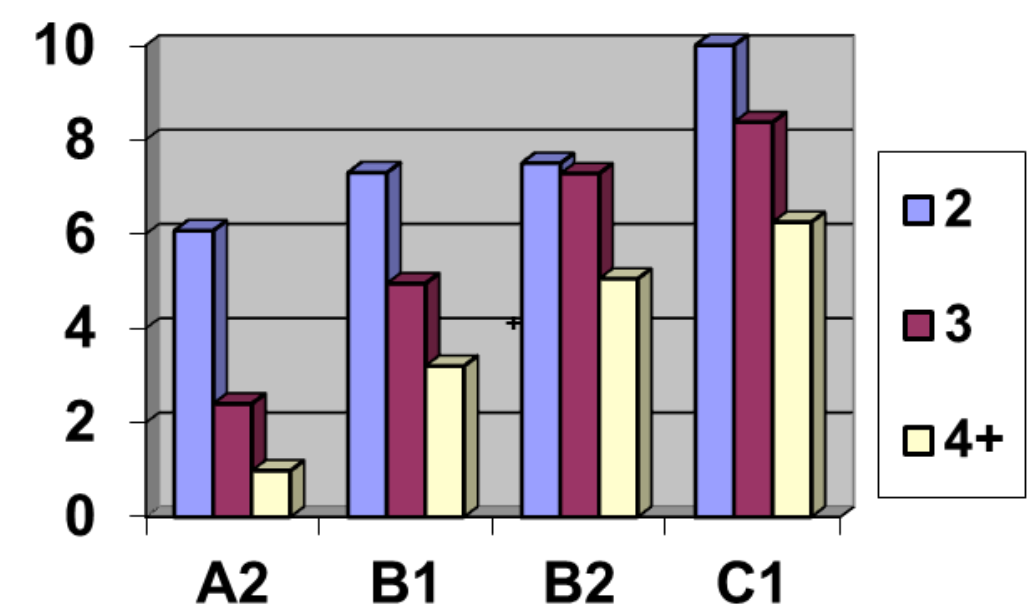

Figura 2. Comparación grado de dificultad y nivel MCER en el test I en traducción directa

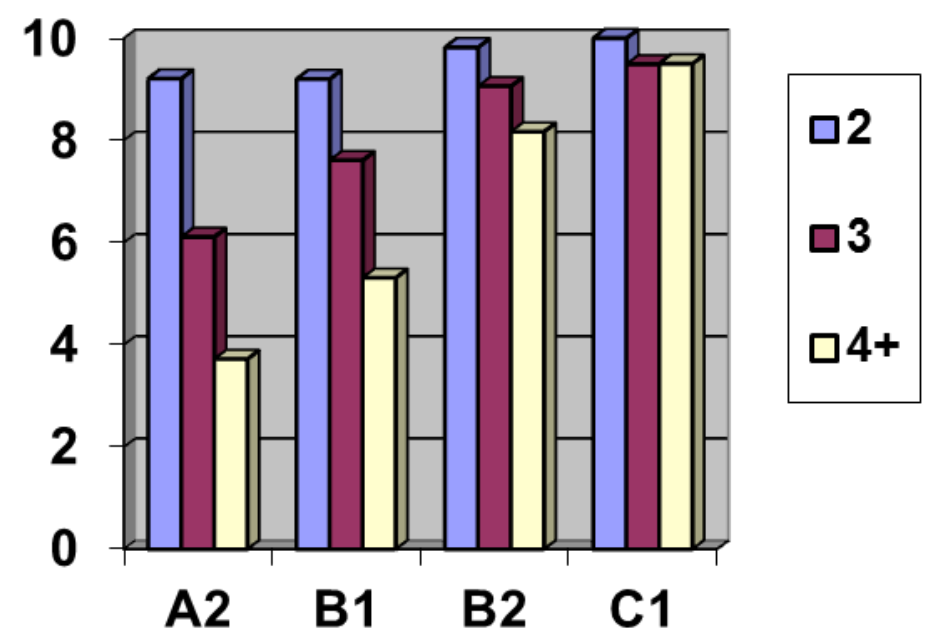

Figura 3. Comparación grado de dificultad y nivel MCER en el test F en traducción directa

El análisis comparativo de los resultados contenidos en las figuras 2 y 3 -test I y test F- revelan que la mejora en el test final es directamente proporcional a la complejidad de los GNs. Es decir que cuanto mayor es el número de elementos del GN, mayor es el incremento de mejora, ya que los resultados iniciales eran más bajos por el desconocimiento general de 
los GNs de 3 y más elementos. En el grado de dificultad 2 se observan las menores mejoras en comparación con los GNs complejos.

Es importante destacar la diferencia entre el usuario competente $\mathrm{C} 1$ y el resto de los niveles MCER. El usuario competente muestra a la vez una puntuación final más alta y un incremento menor de mejora en GNs de 2 y 3 elementos, que ya los conocía inicialmente. En cuanto al grado de dificultad 3, la mejora aumenta gradual e inversamente al nivel MCER de los alumnos. En el grado de dificultad 4+, es de destacar la mejora de los alumnos B2 y C1.

\section{Traducción Inversa: Análisis por grado de dificultad}

El grado de dificultad de la traducción inversa de los GNs viene determinado por el número de elementos que componen cada GN. La tabla 9 muestra la puntuación media sobre 10, por grado de dificultad en el test I y la mejora en el test F, sin distinguir niveles MCER.

Tabla 9. Medias por dificultad en el test I de traducción inversa y de mejoras en test $\mathrm{F}$

\begin{tabular}{cccc} 
DIFICULTAD & NoALUMNOS & $\begin{array}{c}\text { PUNTUACIÓN } \\
\text { MEDIA TEST I. }\end{array}$ & $\begin{array}{c}\text { MEJORA MEDIA } \\
\text { EN TEST F. }\end{array}$ \\
\hline 2 & 50 & 6,49405 & 1,55357 \\
\hline 3 & 50 & 4,42163 & 2,26339 \\
\hline $4+$ & 50 & 2,63818 & 4,20417
\end{tabular}

En esta tabla observamos que en los GNs de 2 elementos, la mejora es de 1,55 puntos sobre la puntuación del test I. Esta va aumentando progresivamente en los GNs de mayor dificultad, ya que la puntuación inicial era inferior, hasta llegar a 4,2 puntos en el caso de los GNs de 4+ elementos.

De manera gráfica (Figura 4) podemos mostrar la mejora global de los participantes, según el grado de dificultad de los GNs.

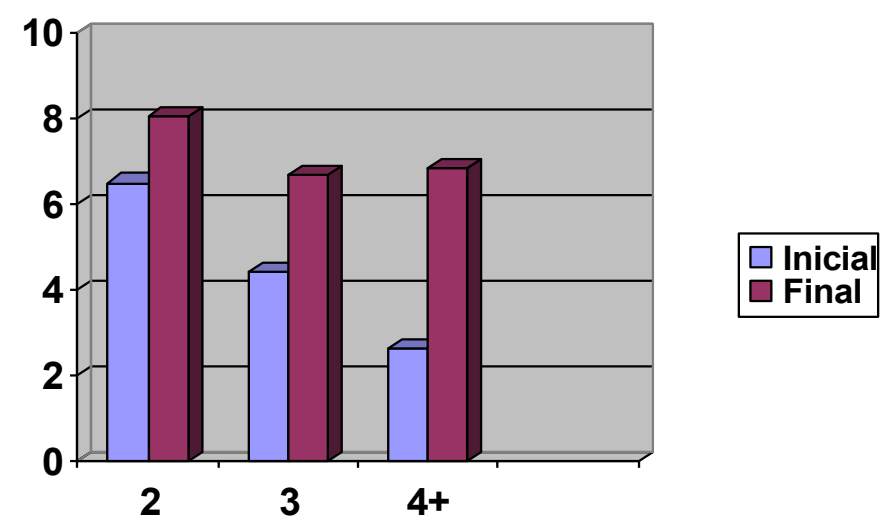

Figura 4. Comparación Test I y Test F por grado de dificultad en traducción inversa 


\section{Traducción inversa: Análisis por nivel MCER}

La tabla 10 recoge las puntuaciones medias del test inicial según el nivel MCER de los participantes, sin distinción del grado de dificultad de los GNs.

Tabla 10. Medias en test I de traducción inversa por nivel MCER

\begin{tabular}{ccc} 
NIVEL & N $^{\text {ALUMNOS }}$ & PUNTACIÓN MEDIA \\
\hline A2 & 7 & 2,4898 \\
\hline B1 & 21 & 3,6523 \\
\hline B2 & 14 & 5,3424 \\
\hline C1 & 8 & 6,5873 \\
\hline
\end{tabular}

Los alumnos de niveles A2 y B1 muestran grandes dificultados para la traducción al inglés de GNs y su media es de 2,4 y 3,6 respectivamente. Los niveles B2 y C1 obtienen medias respectivas de 5,3 y 6,5 muy alejadas de las puntuaciones obtenidas por estos niveles en la traducción directa (Tabla 7). Para poder valorar las diferencias entre los distintos niveles MCER y su comportamiento respecto al grado de dificultad de los GNs, analizamos las puntuaciones de cada nivel en el test inicial por grado de dificultad (Tabla 11).

Tabla 11. Medias por dificultad y por nivel en test I en traducción inversa y de mejora en test $\mathrm{F}$

\begin{tabular}{|c|c|c|c|c|}
\hline DIFICULTAD & $\begin{array}{l}\text { NIVEL } \\
\text { MCER }\end{array}$ & NoALUMNOS & $\begin{array}{l}\text { PUNTUACIÓN } \\
\text { MEDIA TEST I }\end{array}$ & $\begin{array}{l}\text { MEJORA MEDIA } \\
\text { EN TEST F }\end{array}$ \\
\hline 2 & $\mathrm{~A} 2$ & 7 & 3,7143 & 1,7142 \\
\hline 2 & $\mathrm{~B} 1$ & 21 & 5,9047 & 2,0 \\
\hline 2 & $\mathrm{~B} 2$ & 14 & 7,8571 & 1,0 \\
\hline 2 & $\mathrm{C} 1$ & 8 & 8,50 & 1,5 \\
\hline 3 & A2 & 7 & 3,1428 & 1,6 \\
\hline 3 & $\mathrm{~B} 1$ & 21 & 3,5555 & 2,4714 \\
\hline 3 & $\mathrm{~B} 2$ & 14 & 4,9047 & 2,65714 \\
\hline 3 & $\mathrm{C} 1$ & 8 & 6,0833 & 2,325 \\
\hline $4+$ & $\mathrm{A} 2$ & 7 & 0,6122 & 4,7 \\
\hline $4+$ & $\mathrm{B} 1$ & 21 & 1,4966 & 4,37 \\
\hline $4+$ & $\mathrm{B} 2$ & 14 & 3,2653 & 4,25 \\
\hline $4+$ & $\mathrm{C} 1$ & 8 & 5,1785 & 3,25 \\
\hline
\end{tabular}

Observamos que todos los alumnos mejoran sus puntuaciones en el test final de traducción inversa de GNs con distintos grados de complejidad y que cuanto mayor es la dificultad, mayor es el grado de mejora en todos los niveles porque partían de un menor conocimiento. El nivel A2 es el que experimenta una mejora mayor en prácticamente todos los grados de dificultad de los GNs, especialmente en el de 4+ elementos.

El índice de mejora de los alumnos de los niveles B1 y B2 es muy similar para los GNs complejos de 3 y $4+$ elementos. La mejora del nivel $\mathrm{C} 1$ es progresiva si bien menor que en el resto de los niveles. Gráficamente, los resultados quedan recogidos en las figuras 5 y 6 . 


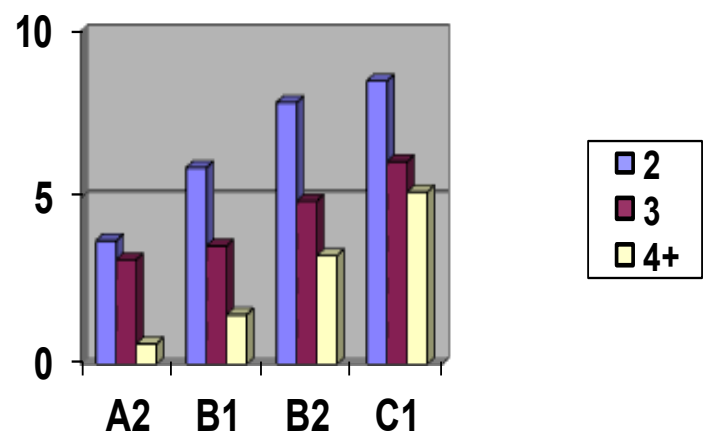

Figura 5. Comparación grado de dificultad y nivel MCER test I en traducción inversa

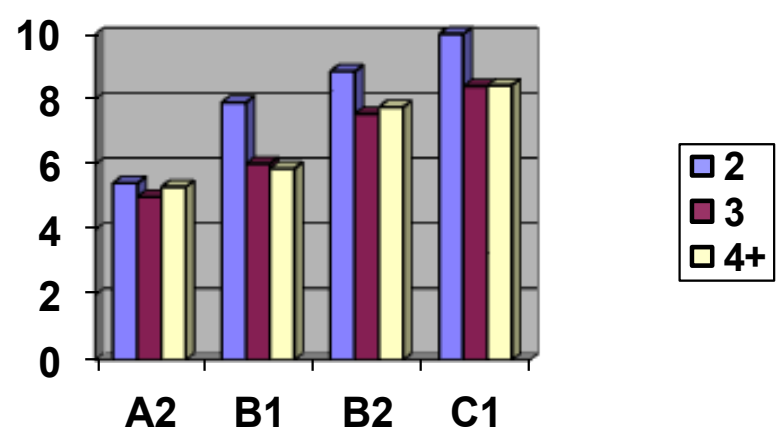

Figura 6. Comparación grado de dificultad y nivel MCER test $\mathrm{F}$ en traducción inversa

La Tabla 12 ofrece a modo de resumen los resultados de los datos obtenidos en este estudio empírico, tanto en traducción directa como inversa, por niveles MCER y por complejidad del GN en cuanto al número de elementos premodificadores que lo forman.

Tabla 12. Resumen de resultados en traducción directa e inversa

TRADUCCIÓN DIRECTA

\begin{tabular}{|c|c|c|c|c|}
\hline Nivel & Dificultad & Test I & Mejora & Test F \\
\hline \multicolumn{5}{|l|}{ A2 } \\
\hline & 2 & 6,07 & 2,14 & 8,21 \\
\hline & 3 & 2,43 & 3,71 & 6,14 \\
\hline & $4+$ & 0,99 & 2,74 & 3,73 \\
\hline \multicolumn{5}{|l|}{ B1 } \\
\hline & 2 & 7,38 & 1,9 & 9,28 \\
\hline & 3 & 4,95 & 2,66 & 7,61 \\
\hline & $4+$ & 3,30 & 2,12 & 5,42 \\
\hline \multicolumn{5}{|l|}{$\mathrm{B} 2$} \\
\hline & 2 & 7,5 & 2,32 & 9,32 \\
\hline & 3 & 7,29 & 1,78 & 9,07 \\
\hline & $4+$ & 5,05 & 3,12 & 8,17 \\
\hline $\mathrm{C} 1$ & & & & \\
\hline
\end{tabular}

TRADUCCIÓN INVERSA

\begin{tabular}{|c|c|c|c|}
\hline Dificultad & Test I & Mejora & Test F \\
\hline \multicolumn{5}{|l|}{} & & \\
\hline 2 & 3,71 & 1,71 & 5,42 \\
\hline 3 & 3,14 & 1,60 & 5,00 \\
\hline $4+$ & 0,61 & 4,70 & 5,31 \\
\hline \multicolumn{4}{|l}{} \\
\hline 2 & 5,90 & 2,00 & 7,90 \\
\hline 3 & 3,55 & 2,47 & 6,02 \\
\hline $4+$ & 1,50 & 4,37 & 5,87 \\
\hline \multicolumn{4}{|l|}{} \\
\hline 2 & 7,86 & 1,00 & 8,86 \\
\hline 3 & 4,90 & 2,66 & 7,56 \\
\hline $4+$ & 3,26 & 4,50 & 7,75 \\
\hline \multicolumn{4}{|l}{} \\
\hline
\end{tabular}




\begin{tabular}{|c|c|c|c|c|}
\hline & 2 & 10 & 0,0 & 10,00 \\
\hline & 3 & 8,37 & 1,25 & 9,62 \\
\hline & $4+$ & 6,25 & 3,25 & 9,50 \\
\hline
\end{tabular}

\begin{tabular}{|c|c|c|c|}
\hline 2 & 8,50 & 1,50 & 10,00 \\
\hline 3 & 6,08 & 2,32 & 8,40 \\
\hline $4+$ & 5,18 & 3,25 & 8,43 \\
\hline
\end{tabular}

\section{Conclusiones}

A la vista del análisis estadístico de los datos con un nivel de confianza superior al $95 \%$ establecido para el estudio, la primera conclusión es que existe una alta relación entre la enseñanza de la estructura sintáctica de los GNs y el nivel de mejora en su conocimiento por parte de todos los participantes con niveles MCER de A2 a C1.

En cuanto a la receptividad al aprendizaje de los GN en ICT, según los niveles MCERL de los participantes, podemos afirmar lo siguiente:

El nivel A2 es el único que registra una puntuación menor al 50\% de aciertos en traducción directa de GN con $4+$ elementos $(3,73)$ después del proceso enseñanzaaprendizaje. En traducción inversa de GN con $4+$ elementos supera el 50\% (5,31), al experimentar una mejora de 4,7 puntos, lo que resulta sorprendente desde el punto de vista lingüístico, por la mayor dificultad que suele representar la traducción inversa frente a la directa y porque ninguno de los resultados iniciales de este grupo alcanzaba $50 \%$ en traducción inversa. También este ha sido el único valor $\mathrm{P}>0,05$, que no concuerda exactamente con la expectativa del estudio, aunque el desvío no ha sido grande $(\mathrm{P}=0,11)$. El aprendizaje de los GN en traducción directa ha podido surtir un efecto potenciador en el aprendizaje posterior de traducción inversa, tanto en este caso como en el siguiente nivel B1. El nivel B1 supera el 50\% de aciertos en todos los casos tanto en traducción directa como inversa, si bien en los GN de 4+ elementos no llega al 60\%, como sí lo hacen los niveles superiores. El nivel B2 supera el $80 \%$ de aciertos en traducción directa e inversa de 2 elementos y el $75 \%$ en inversa de 3 y $4+$ elementos. Finalmente, el nivel C1 supera el 95\% en traducción directa y el $80 \%$ en inversa, perfeccionando sus buenas puntuaciones de partida.

A la vista de los resultados, se puede afirmar que los participantes con niveles B2 y $\mathrm{C}$, una vez superada la fase de enseñanza-aprendizaje, obtienen un alto índice de aciertos en las pruebas finales que nos permitirían hablar de la adquisición de este rasgo lingüístico. En el caso del nivel B2 se debe claramente a su aprendizaje (pasan de $49 \%$ aciertos a $76 \%$ en directa y de $32,5 \%$ a $77,5 \%$ de aciertos en inversa).

En los niveles A2 y B1 habría que realizar pruebas de seguimiento para saber si la estructura se ha adquirido o si los resultados reflejan exclusivamente el momento de enseñanza, sin una completa asimilación de la estructura. 
Vemos, por lo tanto, que para adquirir la competencia gramatical en el uso de los GNs, incluso tras la exposición a un proceso intensivo de enseñanza-aprendizaje, se requiere un nivel del MCER igual o superior a B2. Este estudio viene a apoyar la posición de que para la enseñanza de una estructura compleja no existente en la lengua materna, ésta solo se asimila cuando el usuario parte de unas competencias lingüísticas adecuadas para la asimilación del nuevo rasgo lingüístico. Esto tiene especial trascendencia para la programación docente del ICT cuyo objetivo último es alcanzar la competencia lingüística como requisito para la competencia comunicativa en los entornos académicos y profesionales. También tiene especial relevancia para la evaluación, ya que no se puede exigir el uso correcto de una estructura para la cual el alumno no está preparado.

\section{Referencias}

Alcaraz, E. (2000). El inglés profesional y académico. Madrid. Alianza Editorial.

Bocanegra Valle, A. (2007). On the teachability of nominal compounds to Spanish learners of English for specific purposes. En A. Bocanegra Valle, M.C. Lario de Oñate y López E. Torres (Eds.) English for specific purposes: Studies for classroom development and implementation (pp. 73-94). Cádiz: Servicio de publicaciones de la Universidad de Cádiz.

Brown G., Malmkjaer, K., \& Williams J. (1996). Performance and Competence in Second Language Acquisition. Cambridge: Cambridge University Press.

Cademártori, Y., Parodi, G., \& Venegas, R. (2006). El discurso escrito y especializado: caracterización y funciones de las nominalizaciones en los manuales técnicos. Literatura y Lingüística, 17, 243-265.

Consejo de Europa (2001). Marco común europeo de referencia para las lenguas: aprendizaje, enseñanza, evaluación. Madrid: Instituto Cervantes.

Downing, A. (1997). On the creation and use of English compound nouns, Language, 53, 810-842.

Dudley-Evans, T. (2001). English for specific purpose. En R. Carter \& D. Nunan (Eds.), The cambridge guide to teaching English to speakers of other languages (pp. 131-136). Cambridge. Cambridge University Press.

Durán Escribano, P. (2004). Developing language awareness in EST students: Teaching principles and strategies. En S. Barrueco, E. Hernández, E. \& L. Sierra (Eds.). VIII jornadas de lenguas para fines específicos. Investigación y enseñanza (pp. 185-189). Alcalá de Henares: Universidad de Alcalá de Henares.

Durán Escribano, P., \& Rubio, A.L. (2015). Language proficiency level and intake of nominal groups use in scientific English: a web classroom empirical study. International Journal of English Linguistics, Vol 5(2): 21-35.

Ellis, R. (1984). Can syntax be taught? A study of the effects of formal instruction acquisition. Applied Linguistics 5: 138-55.

Giovannini, A., Martín Peris, E., Rodríguez, M. y Simón, T. (1996). Profesor en acción: vol. 1: El proceso de aprendizaje. Vol. 2: Áreas de trabajo. Vol. 3: Destrezas. Madrid: Edelsa.

Girju, R., Moldovan, D., Tatu, M. y Antohe D. (2005). On the semantics of noun compounds. Computer Speech and Language, 19: 479-496. 
Halliday, M., \& Martin, J. (1993). Writing science: Literacy and discourse power. Londres: Falmer Press.

Huckin, T.N., \& Olsen, A. (1991). Technical writing and professional communication for nonnative speakers of English. New York: McGraw-Hill.

Norman, G. (1999). Cómo escribir un artículo científico en inglés. Madrid: Astrazeneca.

Martínez, N., \& Hurtado, A. (2003). An assessment in translation studies: research needs. Meta XLVI, 2. Disponible en http://erudit.org/revue/meta.

Pastor Cesteros, S. (2004). Aprendizaje de segundas lenguas. Lingüística aplicada a la enseñanza de idiomas. Alicante: Publicaciones de la Universidad de Alicante.

Rubio Moreda, A. L. (2015). La competencia lingüística en el uso de los grupos nominales en inglés académico y profesional: estudio empírico. Madrid. Universidad Politécnica de Madrid. (tesis doctoral inédita).

Schmidt, R. (2001). Attention. En P. Robinson (Ed.) Cognition and second language instruction (pp. 3-32). Cambridge. Cambridge University Press.

Swales, J.M. (1985). Episodes in ESP. A source and reference book in the development of English for science and technology. Oxford: Pergamon Press.

Trimble, L. (1985). English for Science and Technology: A Discourse Approach. Cambridge. Cambridge University Press.

\section{Referencia de la autora}

Ana Luz Rubio Moreda es es Doctora (2015) y profesora del Departamento de Lingüística Aplicada a la Ciencia y la Tecnología de la UPM. Actualmente coordina la asignatura English for Professional and Academic Communication en el Grado de Tecnologías Industriales. Su línea de investigación incluye lingüística aplicada a ICT y nuevas metodologías en enseñanza.

Email: analuz.rubio@,upm.es

Para citar este artículo:

Rubio Moreda, A.L. (2015). Receptividad al aprendizaje de grupos nominales en inglés según el nivel MCERL. Bellaterra Journal of Teaching \& Learning Language \& Literature, 8(3), 78-93. DOI: http://dx.doi.org/10.5565/rev/jt13.603 